\title{
Engineering Local Fields in Nonlinear Plasmonic Metasurfaces -INVITED
}

\author{
Mikko J. Huttunen1,*, Saad Bin-Alam2, Orad Reshef,, Yaryna Mamchur4, Timo Stolt1, Jean-Michel Ménard3, \\ Ksenia Dolgaleva2,3, Robert W. Boyd2,3,5 and Martti Kauranen1 \\ 1Photonics Laboratory, Physics Unit, Tampere University, Tampere, Finland \\ ${ }_{2}$ School of Electrical Engineering and Computer Science, University of Ottawa, Ottawa, ON, K1N 6N5, Canada \\ 3Department of Physics, University of Ottawa, Ottawa, ON, K1N 6N5, Canada \\ 4National Technical University of Ukraine "Igor Sikorsky Kyiv Polytechnic Institute," Kyiv, Ukraine \\ ${ }_{5}$ The Institute of Optics and Department of Physics and Astronomy, University of Rochester, Rochester, New York 14627, USA
}

\begin{abstract}
Nonlinear optical phenomena are paramount in many photonic applications ranging from frequency broadening and generation of ultrashort pulses to frequency comb-based metrology. A recent trend has been to miniaturize photonic components, resulting also in a demand for small scale nonlinear components. This demand is difficult to address by using conventional materials motivating the search for alternative approaches. Nonlinear plasmonic metasurface cavities have recently emerged as a promising platform to enable nanoscale nonlinear optics. Despite steady progress, the so far achieved conversion efficiencies have not yet rivalled conventional materials. Here, we discuss our recent work to develop more efficient nonlinear metamaterials, focusing on plasmonic metasurfaces supporting collective responses known as surface lattice resonances. These resonances can exhibit very narrow spectral features, showing potential to considerably enhance nonlinear processes via resonant interactions. We demonstrate a plasmonic metasurface operating at the telecommunications $C$ band that exhibits a record-high quality factor close to 2400, demonstrating an order-of-magnitude improvement compared to existing metasurface cavities. Motivated by this experimental demonstration, we also present numerical predictions suggesting that such metasurfaces could soon answer the existing demand for miniaturized and/or flat nonlinear components.
\end{abstract}

\section{Introduction}

Recent investigations have shown that periodic arrays of nanoparticles, also known as metasurfaces, can act as optical cavities [1]. Major challenge in metasurface cavities has so far been their poor cavity lifetimes hindering their use in potential applications. For example, the resonances associated with individual plasmonic nanoparticles known as localized surface plasmon resonances (LSPRs) are associated with low $(<10)$ quality factors ( $Q$-factors). Already around 10-fold improvements in $Q$-factors have been recently demonstrated using dielectric nanoparticle resonators [2], while dramatically higher $Q$-factors have been predicted to be possible by utilizing collective responses known as surface lattice resonances (SLRs) [3,4]. However, currently demonstrated $Q$-factors have been so far limited to few hundreds [6].

Here, we demonstrate a plasmonic metasurface cavity design supporting an ultra-high- $Q$ SLR $(Q \approx 2400)$ near $\lambda=1545 \mathrm{~nm}$ [5]. Inspired by this result, we will also use nonlinear discrete-dipole approximation (NDDA) to numerically investigate second-harmonic generation (SHG) from such high- $Q$ metasurfaces [7]. Over a million-fold SHG intensity enhancement is predicted.

\section{Results for high-Q metasurfaces}

Theory providing an intuitive and qualitative agreement with the experimental results is based on lattice sum calculations and has been described elsewhere [3,8]. Here, the lattice sum approach (LSA) was used to design a high$Q$ metasurface by arranging rectangular gold nanobars (lengths $L_{y}=200 \mathrm{~nm}$, widths $L_{x}=130 \mathrm{~nm}$ and heights $t=20 \mathrm{~nm}$ ) into a rectangular array with periodicities of $p_{x}=500 \mathrm{~nm}$ and $p_{y}=1060 \mathrm{~nm}$, immersed in a homogeneous environment with refractive index of $n=$ 1.45 (see Fig. 1a). The area of the fabricated metasurface was $600 \times 600 \mu \mathrm{m}^{2}$, consisting of $\approx 1200 \times 567$ particles. Based on finite-difference time domain (FDTD) simulations the designed metasurface was expected to support a LSPR near $850 \mathrm{~nm}$ and a high- $Q$ SLR near 1550 $\mathrm{nm}$. The measured transmission spectra (see Fig. 1b), matches very well the calculated spectra. Most notably, the full width at half-maximum of the linewidth of the SLR is only $\Delta \lambda=0.66 \mathrm{~nm}$, corresponding to a recordhigh quality factor of $Q=2400$ for a plasmonic device.

\section{Multiresonant high-Q metasurfaces for nonlinear optics}

Then we generalize the LSA to describe nonlinear responses and finite arrays [9-11], motivated to investigate metasurfaces that could support multiple high$Q$ SLRs. By designing such metasurfaces to exhibit high$Q$ resonances, not just near the fundamental pump frequency, but also near the signal frequencies of interest, multiresonant nonlinear materials for enhanced frequency conversion could be realized $[8,12]$. The extension of the LSA to finite arrays and arbitrary excitation field profiles is done by using nonlinear discrete-dipole approximation (NDDA), which takes explicitly into account the scattered fields from every particle in the metasurface $[7,10]$.

\footnotetext{
* Corresponding author: mikko.huttunen@,uni.fi
} 


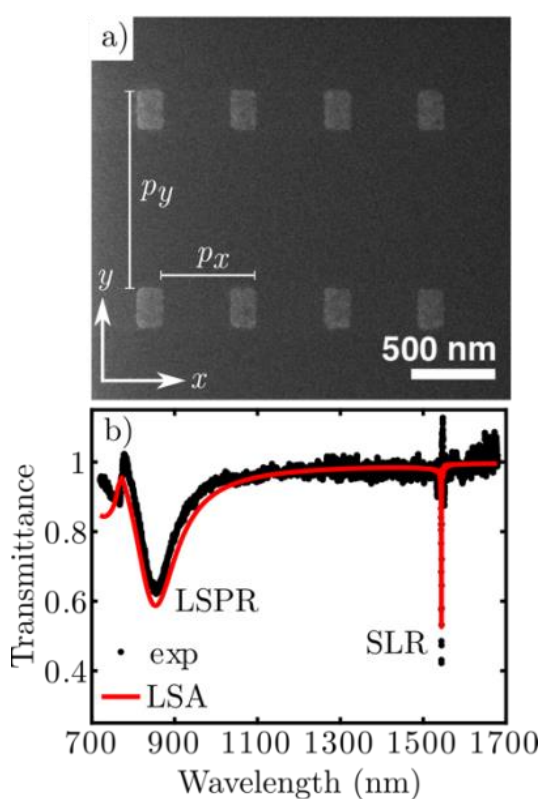

Fig. 1. a) Helium-ion micrograph of the fabricated metasurface. b) Measured (black) and calculated (red) transmission spectra showing the LSPR $(850 \mathrm{~nm})$ and SLR $(1535 \mathrm{~nm})$. The SLR peak at the telecommunications $C$ band exhibits an ultra-narrow linewidth of $0.66 \mathrm{~nm}$, corresponding to $Q \approx 2400$.

Here, we introduce a metasurface design consisting of asymmetric L-shaped aluminum nanoparticles with thicknesses $h=30 \mathrm{~nm}$, left-side arm length $l_{1}=100 \mathrm{~nm}$, right-side arm length $l_{2}=90 \mathrm{~nm}$ and arm width of $w=$ $45 \mathrm{~nm}$ (Fig. 2a). Although gold is a typical plasmonic material, we were not able to find particle dimensions that would have supported LSPRs at short enough wavelengths. Subsequently, aluminium nanoparticles were used which allowed the shorter wavelength LSPR to peak near $470 \mathrm{~nm}$, supporting emergence of high- $Q$ SLRs at longer wavelengths [10]. The nanoparticles were arranged into a rectangular lattice with the periods $p_{x}=$ $395 \mathrm{~nm}$ and $p_{y}=793 \mathrm{~nm}$, giving rise to the two high- $Q$ SLRs at the second-harmonic $(600 \mathrm{~nm})$ and the fundamental wavelengths $(1200 \mathrm{~nm})$, respectively (Fig. 2b). Notably, over a million-fold intensity enhancement of SHG emission was predicted to occur when the fundamental and SHG frequencies coincided spectrally with the SLRs (Fig. 2c).

\section{Conclusion}

We have demonstrated an SLR-based plasmonic metasurface nanocavity with a record-high $Q$-factor of 2400. Such high- $Q$ metasurfaces could dramatically expand the current capabilities of metasurfaces. We have also numerically investigated multiresonant metasurfaces, which could enable applications benefiting of multiple high- $Q$ resonances. We believe that such multiresonant high- $Q$ metasurfaces show promise for nonlinear optical applications such as for frequency conversion.

We acknowledge the support of the Academy of Finland (Grant No. 308596) and the Flagship of Photonics Research and Innovation (PREIN) funded by the Academy of Finland (Grant No. 320165)

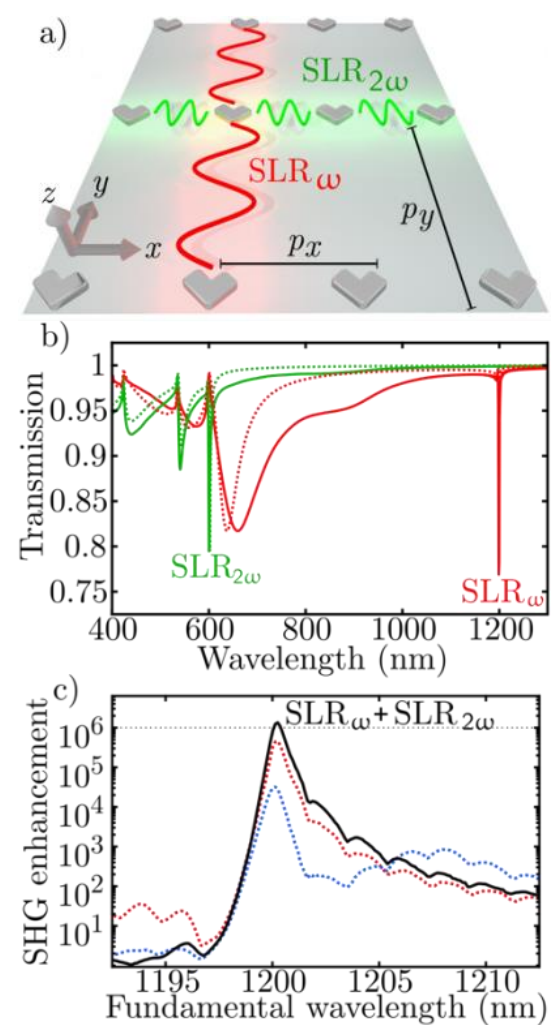

Fig. 2. a) Multiply-resonant metasurface. b) Calculated transmission spectra using the NDDA (dotted lines) and the FDTD (solid lines). Red (green) curves correspond to $x$ polarized ( $y$-polarized) incident light. c) Predicted SHG intensity enhancement for a particle at the center of the metasurface. Over million-fold SHG intensity enhancement occurs for the doublyresonant metasurface (black solid line).

\section{References}

1. N. Meinzer, W. L. Barnes, and I. R. Hooper, Nat. Photonics, 8, 889-898 (2014)

2. K. Koshelev et al., Science, 367, 288-292 (2020)

3. M. J. Huttunen et al., Opt. Express, 24, 28279-28289 (2016)

4. V. G. Kravets et al., Chem. Rev., 118, 5912-5951 (2018)

5. Q. Le-Van, Quynh, et al. Adv. Opt. Mater., 7, 1801451 (2019)

6. M. S. Bin-Alam et al., arXiv:2004.05202[physics.optics] (2020)

7. M. J. Huttunen et al., Phys. Rev. A, 97, 053817 (2018).

8. O. Reshef et al. Nano Lett., 19, 6429-6434 (2019)

9. L. Michaeli et al., Phys. Rev.Lett., 118, 243904 (2017)

10. M. J. Huttunen et al., J. Opt. Soc. Am. B, 36, E30E35 (2019)

11. M. J. Huttunen, R. Czaplicki, and M. Kauranen, J. Nonlinear Opt.Phys. Mater., 28, 1950001 (2019)

12. M. Celebrano et al., Nat. Nanotechnol., 10, 412-417 (2015) 